\title{
USO DE LOS SERVICIOS FINANCIEROS ESTUDIO EMPÍRICO EN ESTUDIANTES UNIVERSITARIOS
}

\author{
Milka Elena, Escalera-Chávez \\ Researcher Professor at Unidad Académica Multidisciplinaria Zona Media. \\ UASLP Rioverde S.L.P. México. \\ milkaech@uaslp.mx \\ Esmeralda Tejada-Peña \\ Researcher Professor at Instituto Tecnológico de Tuxtepec \\ esme_tejada@hotmail.com \\ Arturo, García-Santillán \\ Researcher Professor at UCC Business School \\ Universidad Cristóbal Colón \\ arturogarciasantillan@yahoo.com.mx
}

https://doi.org/10.17060/ijodaep.2017.n1.v2.854

Fecha de recepción: 11 Enero 2017

Fecha de admisión: 1 Abril 2017

\section{RESUMEN}

La población incluida financieramente accede a los servicios bancarios, de ahí que ésta inserción favorece el desarrollo económico de la población, sin embargo existen muchas personas que no utilizan los servicios financieros formales, incluyendo a los estudiantes. Por este motivo, el objetivo de este trabajo fue identificar la frecuencia con la que los estudiantes de nivel superior de Tuxtepec Oaxaca acceden a los servicios financieros que ofrece las Instituciones Bancarias. La muestra está conformada por 800 alumnos del nivel superior que pertenecen a 8 universidades públicas y privadas de Tuxtepec Oaxaca. Se comprobó por medio de la prueba t la frecuencia del uso de los servicios financieros. Los resultados muestran que los estudiantes usan los servicios financieros que ofrecen las Instituciones Bancaria. Sin embargo, es importante reiterar que algunos estudiantes no recurren al sistema financiero, siendo esta proporción de la población un área de oportunidad para el proceso de inclusión financiera en México.

Palabras Claves: Uso, Servicios Financieros, Estudiantes Universitarios. 


\section{INTRODUCCIÓN}

La inclusión financiera es uno de los factores que contribuyen al crecimiento económico y el desarrollo de un país porque su incremento no sólo acelera el desarrollo y el empleo, sino que también enmienda la desigualdad de ingresos y contribuye a la reducción de la pobreza (Alianza para la Inclusión Financiera, 2013). De igual forma el Subgobernador del Banco de México, Manuel Sánchez González han señalado que "la teoría y la experiencia internacional han corroborado con claridad que la inclusión financiera contribuye al desarrollo económico y favorece a la población. En particular, el acceso a los servicios bancarios permite a los individuos y las empresas cuidar su patrimonio, realizar eficientemente transacciones económicas, tomar decisiones de ahorro y financiar sus planes de consumo e inversión.".

Recientemente se llevó a cabo el II Foro Internacional de Inclusión Financiera en México (2016), donde la asesora especial del secretario general de las Naciones Unidas hablo sobre inclusión financiera para el desarrollo y menciona que la inclusión financiera en su esencia se enfoca en el desarrollo y empoderamiento humano, su objetivo es proporcionar de forma responsable y sostenible el acceso a servicios financiero de calidad y accesibles para uso de todos (Máxima, 2016). Atendiendo a estas condiciones, la Inclusión Financiera desempeña un papel importante en fomentar el crecimiento equitativo y el cumplimento de las metas de desarrollo como son la reducción de la pobreza y la seguridad alimentaria, proporcionando herramientas como: el ahorro, el seguro, los pagos, el crédito, a las familia para que puedan estar mejor preparas para los eventos inesperados, aumentar su residencia y reducir los niveles de pobreza, también los pequeños negocios pueden convertirse en empresas de mayor tamaño y con ello las sociedades y las economías pueden crecer, fortalece y tornarse más inclusivas.

Sin embargo a pesar que la inclusión financiera puede contribuir a las mejora económicas y sociales de un país, existen dos mil millones de los adultos en el mundo que no utilizan servicios financieros formales y, más de la mitad de los adultos de más escasos recursos aún no tenían cuentas bancaria debido a los altos costos, las distancias de viaje y a los complejos requisitos que se deben cumplir para abrir una cuenta bancaria. Estudios realizados por Lusardi y Mitchell (2006), revelan que muchos hogares de bajos ingresos no están familiarizados con los conceptos económicos más básicos necesarios para tomar decisiones de ahorro e inversión La falta de conocimiento financiero es generalizada tanto en jóvenes como en las personas de edad avanzada, en los Estados Unidos y otros países no están informados sobre cálculos financieros básicos, y esto ocasiona desconocimiento para realizar ahorros, o bien para formalizar planes para la jubilación, hipotecas y otras decisiones. En Japón un estudio realizado por la universidad Osaka demostró que el $50 \%$ carecía de conocimiento de productos financieros (Herrera y Raccanello, 2014).

En México, en el año 2008 se aplicó una encuesta realizado por la UNAM y Banamex (2008), sobre la cultura financiera de los jóvenes en México, y los resultados revelaron que sólo el $18.5 \%$ de los mexicanos realizan algún tipo de planeación y presupuesto de sus recursos, además reveló que sólo el $3 \%$ de la población mexicana de entre 15 y 29 años de edad cuenta con una inversión bancaria y apenas un $46 \%$ lleva algún registro personal de sus finanzas, mientras que un $52 \%$ ahorra regularmente alguna parte de sus ingresos. En el año 2010, la Comisión Nacional Bancaria y de Valores (CNBV) expresó que el $60 \%$ de la población utiliza los servicios financieros y sólo el $49 \%$ conoce y sabe cómo utilizarlos de forma correcta, ya que desconocen los beneficios que dan los 
instrumentos del sistema bancario y sociedades de inversión, lo cual refleja la carencia de la cultura financiera.

El Banco Mundial en el año 2013, en coordinación sus contrapartes de la CNBV y la CONDUSEF presenta los resultados de la encuesta aplicada a la población de 18 años en adelante, en el año 2012, en dicha encuesta se recopilaron datos sobre prácticas de administración diaria del dinero, planificación financiera, información y elección de productos financieros, actitudes y conocimientos financieros, y características sociodemográfica; dichas variables permiten conocer el comportamientos, actitudes y conocimientos financieros de los Mexicanos, los resultados muestran que el $49 \%$ de los encuestados no utilizan los servicios financieros (formales o informales) y el $51 \%$ que utiliza los servicios financieros lo hace a través del ahorro (Reddy, Bruhn y Tan, 2012).

En el año 2015, se presenta la edición 2014 de la base de datos global de la Inclusión Financiera (Global Findex), puesta en marcha por el Banco Mundial en 2011 proporciona indicadores de 143 países (entre ellos México), con preguntas relacionadas el tema financieros como: ahorro, tarjetas (TDD y TDC), crédito, medios de pagos, canales de acceso, banca móvil, seguros, así como: características demográficas, administración de los gasto, remesas, los resultados dejan ver que en México el nivel de inclusión financiera (medido a través del acceso a cuentas) se encuentra por debajo de la línea de tendencia, lo cual indica está por debajo de su valor esperado.

En lo que respecta al estado de Oaxaca, la CNBV (2010), en su segundo reporte de inclusión financiera, informa que Oaxaca es el estado con menos sucursales Bancarias, pues sólo alrededor del $10 \%$ de sus municipios cuentan con sucursales bancarias, mientras que el promedio nacional se ubica en 42\%. En este mismo sentido, el Banco Mundial (2013) desarrolló un estudio sobre el acceso a servicios financieros en la región Sur/Sureste del país, que incluye a los estados de: Campeche, Chiapas, Oaxaca, Quintana Roo, Tabasco, Veracruz y Yucatán.

Los resultados muestran que los estados más pobres como: Chiapas, Oaxaca son los que registran niveles más bajos respecto al acceso de servicios financieros. La Comisión Nacional del Sistema de Ahorro para el Retiro (CONSAR) en el año 2014, aplicó la primera encuesta nacional de trabajadores independientes con el fin de obtener datos respecto a los hábitos de ahorro y consumo encontrándose que los estados de Chiapas, Oaxaca y Guerrero son las entidades federativas con menos cuentas de ahorro para el retiro por cada 10 mil adultos, con 2,393, 2,660 y 3,114 cuentas, respectivamente.

Ahora bien de acuerdo a CNBV (2015), Oaxaca, Zacatecas y Chiapas son los tres estados con el indicador más bajo con respecto al crédito ya que por 10 mil adultos, se han otorgados menos de 4 mil contratos de crédito de la banca múltiple. De acuerdo a la Encuesta Nacional de Inclusión Financiera (ENIF) 2015, realizada por La Secretaría de Hacienda y Crédito Público (SHCP), a través de la Comisión Nacional Bancaria y de Valores (CNBV), en colaboración con el Instituto Nacional de Estadística y Geografía (INEGI), en México de 22.10 millones de adultos, solo el $29 \%$ utilizan los servicios financieros (crédito), ahora bien de 18.9 millones de adultos el $25 \%$ adquiere algún tipo de seguro, en relación al ahorro para el retiro solo el $41 \%$ de adultos tiene una cuenta de ahorro para su retiro.

Billimoria, Penner y Knoote (2013) señala que las personas interesadas en los negocios reconocen que los jóvenes son actores económicos importantes que necesitan estar participando activamente en el desarrollo de políticas, programas y productos financieros destinada al beneficio, esto significa que se debe de dar a todos los jóvenes el conocimiento para tomar decisiones financieras inteligentes, la oportunidad de acumular ahorros, por ello se requiere que los jóvenes tengan una educación financiera. La educación financiera significa la provisión de servicios 
educativos instrucción y/o materiales diseñados para aumentar la conocimientos financieros y habilidades en los jóvenes, este aprendizaje no tendría sentido si los jóvenes no ponen en práctica esos conocimientos, es decir si no hacen uso de los servicios financieros de calidad que sean proporcionados a precios accesibles de una manera conveniente.

Aun cuando, en la última década, la industria bancaria ha mostrado un gran crecimiento en volumen y complejidad, los estudios anteriores muestran que no existe conocimiento, ni tampoco la población en su gran mayoría utiliza los sistemas financieros (Leeladhar 2006). De ahí ha surgido la preocupación por investigar si los jóvenes estudiantes con frecuencia utilizan los servicios bancarios, 0 si la inclusión financiera no ha alcanzado este segmento de la población. Por ello organismos internacionales y nacionales así como Instituciones Académicas en México como la UNAM tienen como objetivo primordial identificar políticas públicas que promuevan la inclusión financiera (Lee, 2011).

En México el 21 de Julio de 2016, se presenta la política Nacional de Inclusión Financiera, en la cual se busca que todos los mexicanos sin distinción alguna puedan tener acceso al sistema financiero, es decir tener una cuenta de ahorro, contratar seguros, obtener un crédito, se trata también de tener diversas maneras de poder realizar pagos por la prestación de distintos servicios de hacer pagos para que las familias sean menos vulnerables, sean menos frágiles, tengan menos riegos de perder su patrimonio o eventualmente de caer en la pobreza, por ello la política Nacional de Inclusión Financiera articula los esfuerzo gubernamentales y los del sector privado para implementar o mejorar la educación financiera que la gente sepa cómo aprovechar las ventajas de estar en el sistema financiero, desde como abrir una cuenta, como usar una tarjeta de crédito o como invertir su dinero, no se trata simplemente de incorporar usuarios al sistema financiero sino de contar con instrumentos que le sean de utilidad y que conozcan además como aprovecharlos, para fomentar esta cultura desde temprana edad la secretaria de educación pública promoverá la Educación Financiera en los planes de estudios de educación básica, así como desarrollando infraestructura financiera en las zonas poco atendidas, mayor acceso y uso de servicios financieros formales a este tipo de población.

De la revisión de los estudios sobre la inclusión financiera, así como del uso y conocimiento sistema financiero, y de las acciones que los diferentes organismos desean realizar para integrar a los sectores de menores recursos y a las empresas al sistema financiero, cabe hacerse la siguiente interrogante: ¿Cuál es la frecuencia con que los estudiantes de nivel superior de Tuxtepec Oaxaca utilizan los servicios financieros que ofrecen las instituciones bancarias? Para dar respuesta se plantea el siguiente objetivo de investigación: Identificar la frecuencia con la que los estudiantes de nivel superior de Tuxtepec Oaxaca acceden a los servicios financieros que ofrece las Instituciones Bancarias.

Siendo los estudiantes uno de los elementos que se convertirá en un ente importante en el desarrollo económico de un país, se requiere conocer, si en algún momento ha hecho uso de los servicios financiero dentro de su ámbito; por tal motivo, la obtención de información y de su análisis contribuirá a identificar a este sector dentro de la inclusión financiera ya que muchos de ellos reciben ingresos por medio de becas o porque realizar una actividad económica y que muchas veces si ahorran, no lo hacen a través de una institución financiera porque no conocen los servicios que ofrecen, o bien 'porque perciben que son muy caros. 
Además, la información será útil para que las instituciones educativas junto con las instituciones financieras deben de proporcionar de manera profesional una educación financiera a los estudiantes según sus expectativas ya que en el futuro serán profesionistas que incursionaran en el mercado laboral. Por ello los organismos educativos y financieros deben asegurase de que los estudiantes conozcan estos servicios y la frecuencia con que recurren a ellos para lograr una mejor incursión financiera.

Con base a lo mencionado anterior, se justifica el análisis de la incursión financiera en estudiantes del nivel superior como base fundamental para reducir la pobreza e incrementar el crecimiento y desarrollo de una región y por ende del país.

\section{REVISIÓN DE LA LITERATURA}

La tendencia de la inclusión financiera está enfocada en ofrecer a los niños y jóvenes el acceso y oportunidad de utilizar los productos y servicios financieros (CDS, 2013). Los niños y jóvenes son considerados competentes financieramente cuando tienen el conocimiento para tomar decisiones financieras inteligentes. Child and Youth Finance International (CYFI) creó una teoría conocida como teoría del cambio (Billimoria, Penner y Knoote, 2013), desarrollada por un grupo de académicos miembros de dicha organización. La teoría combina la educación financiera con la educación social, así como la inclusión financiera, con el fin de aumentar la capacidad financiera y la potenciación socioeconómica de los niños y jóvenes, para conducirlos a una mayor conciencia del entorno financiero (Figura 1).

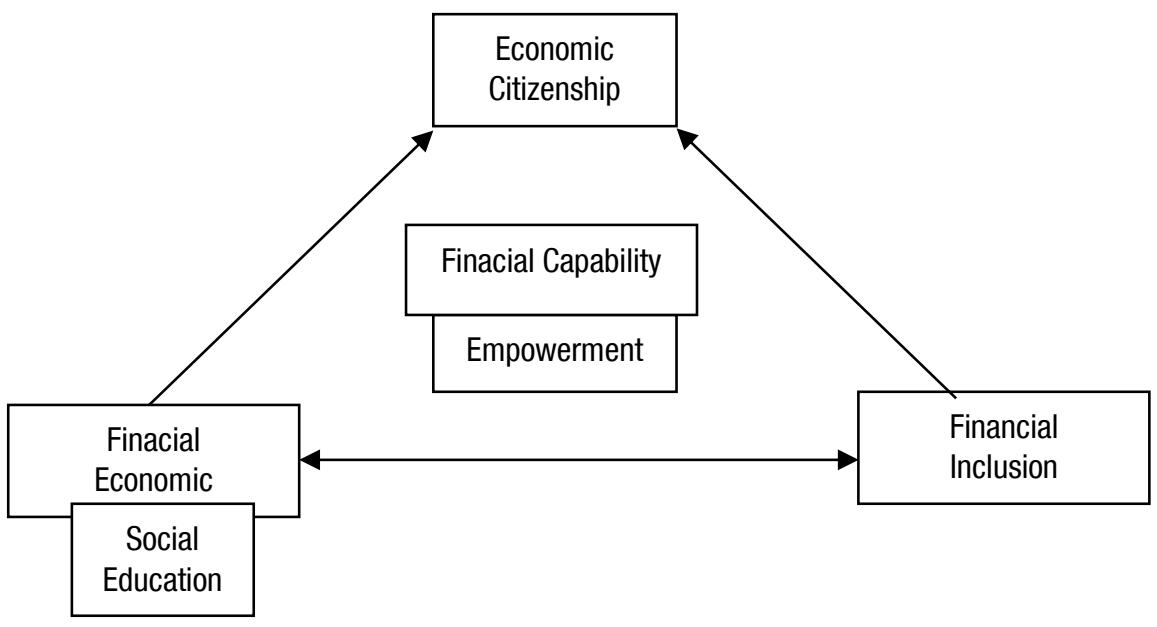

Figure 1 Theory of Change

En el modelo, el grupo de trabajo propuso que la educación financiera, la educación social y la inclusión financiera son los componentes básicos de la potenciación y la capacidad financiera que sustentan en la responsabilidad económica para los niños y jóvenes, obteniendo como resultado reducir la pobreza, mejorar el bienestar económico y social sostenible, y proporcionar medios de 
subsistencia más sostenibles. Aunque están relacionadas, estos términos se utilizan de maneras específicas y distintas en los programas e investigación.

La capacidad financiera es el conocimiento, habilidades financieras y acceso a los servicios financieros, que permiten a la gente a entender y actuar en sus mejores intereses económicos (). La educación financiera incluye la instrucción y/o materiales diseñados para aumentar el conocimiento y las habilidades financieras. La educación social es la prestación de conocimientos y habilidades que cambian la comprensión y el conocimiento de los derechos de los individuos y de los derechos de los demás. Eso también implica el fomento de habilidades para la vida.

La inclusión financiera es apropiada para el acceso, la calidad y accesibilidad a los servicios financieros. La potenciación es la sensación de confianza y eficacia que viven los niños y la juventud mediante el control de sus propias vidas, reclamando sus derechos. Con relación al constructo de inclusión finanicera, se puede medir a través de varias dimensiones, incluida la accesibilidad, asequibilidad, conveniencia, flexibilidad, fiabilidad y seguridad. Como se muestra en la figura 2 .

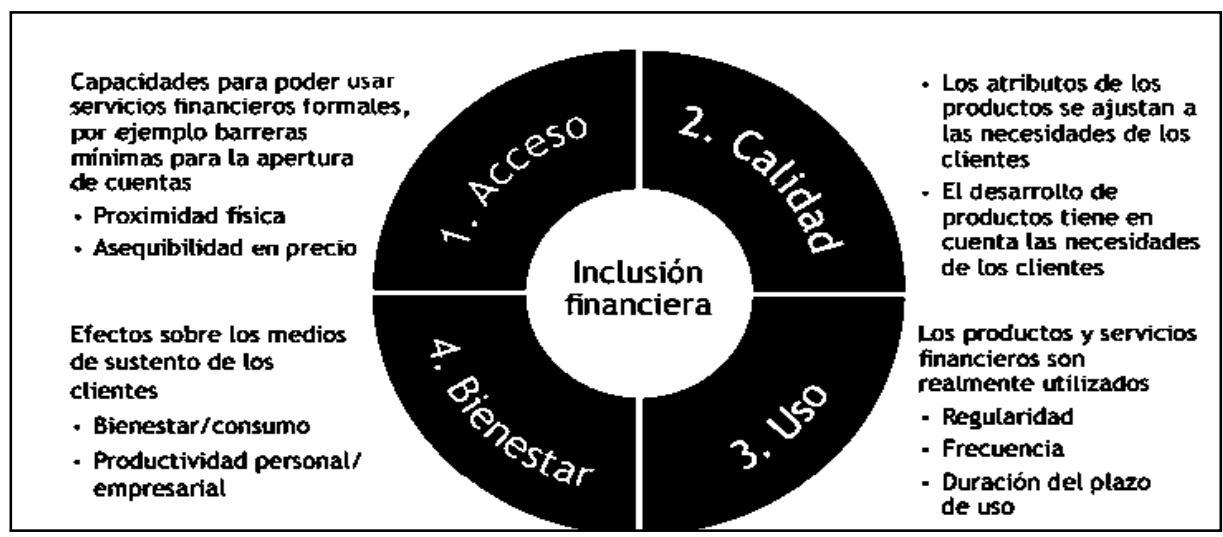

Figura 2. Posibles ópticas a través de las cuales medir la inclusión finanicera

Acceso: referido a la infraestructura financiera disponible para brindar servicios y productos financieros, tales como sucursales, cajeros automáticos, terminales punto de venta, banca móvil y banca por internet.

Uso: se define como la contratación de uno o más productos o servicios financieros, y la frecuencia con que se utilizan; incluyen cuentas de ahorro, de cheques y de nómina, depósitos a plazo, tarjetas de crédito, créditos hipotecarios, automotrices y personales, seguros de vida, de auto, de daños y de gastos médicos, cuentas de ahorro para el retiro, entre otros.

\section{METODOLOGÍA}

El enfoque utilizado en esta investigación es de tipo cuantitativo, en este enfoque el planteamiento epistemológico parte de la unidad del método científico, adoptando el modelo hipotético deductivo. De ahí que se busca probar si los estudiantes de nivel superior de Tuxtepec Oaxaca usan los servicios financieros que ofrecen las instituciones bancarias. 
El diseño de esta investigación es de tipo no experimental, transeccional, exploratorio y causal. No experimental porque no se pueden manipular las variables, los datos a reunir se obtendrán de los estudiantes del nivel superior de Tuxtepec, Oaxaca y Transeccional ya que la recolección de los datos se realiza en un sólo período.

La investigación se circunscribe al estado de 0axaca cuyas características y problemas en el tema de Inclusión Financiera, son semejantes a otros estados del país como: Chiapas, Guerrero, y Michoacán por citar algunos estados. Para el trabajo de campo, se tiene como población focal a ocho Universidades Publicas y Privadas las cuales se ubican en la región de San Juan Bautista Tuxtepec del estado de 0axaca, los cuales cuentan con una población total de 7,668 estudiantes. Para calcular la muestra (366 alumnos a encuestar) se siguió el procedimiento que señala Levin (2002) citado por Moreno-García, García-Santillán y Munguía-Tiburcio (2013), el cual sugiere utilizar la siguiente fórmula:

$$
n=\frac{N Z^{2}(P)(Q)}{e^{2}(N-1)+Z^{2}(p q)}
$$

Se utilizó un cuestionario diseñado con un total de 12 preguntas ( $\alpha$ de Cronbach $=.731$ ) dividido en dos secciones: en la primera sección, las preguntas se relacionaron con el perfil del encuestado, como género, edad, estatus laboral, entre otras y la segunda proporcionara información para investigar el conocimiento que tienen sobre: Presupuesto, Ahorro, Administración de la deuda, Servicios Bancarios, Negociaciones Financiera, Inversión, Crédito, Seguros y Pensiones. Las preguntas fueron acompañadas de una escala de Likert, el rango en esa escala fue desde 1 (siempre) a 5 (nunca).

La recolección de datos se efectuó en dos etapas la primera consistió en aplicar la prueba piloto para validar el instrumento y la segunda fue la aplicación definitiva. La prueba piloto para este estudio se llevó a cabo sobre una muestra compuesta por 30 alumnos de nivel superior de la Universidad Autónoma de San Luís Potosí. Los resultados de la prueba mostraron que las preguntas habían sido claramente formuladas. La segunda etapa, la recolección definitiva se realizó de una manera similar al procedimiento utilizado en la prueba piloto, pero esta vez con alumnos de nivel superior del Municipio de San Juan Bautista Tuxtepec, Oaxaca, posteriormente se procesó la información utilizando el programa SPSS versión 23 .

\section{RESULTADOS}

Como resultado del procedimiento llevado acabo para el análisis e interpretación a los datos obtenidos en campo, se muestra en la tabla 2, algunas de las características distintivas del perfil de los estudiantes que participaron en la investigación. Se observa un alto porcentaje que recibe beca y un porcentaje medio que estudian y trabajo.

Tabla 2 Perfil de los estudiantes

\begin{tabular}{llllll}
\hline $\begin{array}{l}\text { Masculino } \\
(\%)\end{array}$ & $\begin{array}{l}\text { Femenino } \\
(\%)\end{array}$ & \multicolumn{2}{l}{ Beca (\%) } & & $\begin{array}{l}\text { Estudia y trabaja } \\
(\%)\end{array}$ \\
& 54.1 & $\mathrm{Si}$ & No & & \\
\hline
\end{tabular}


En la tabla 3 se muestra la frecuencia con que los estudiantes de nivel superior de Tuxtepec Oaxaca acceden a los servicios financieros que ofrece las Instituciones Bancarias.

Tabla 3. Frecuencia a servicios financieros

\begin{tabular}{c|llc}
\hline No. & Frecuencia & $\begin{array}{l}\% \\
\text { Valido }\end{array}$ & $\begin{array}{c}\% \\
\text { acumulado }\end{array}$ \\
\hline 1 & Siempre & 9.5 & 9.5 \\
2 & Muchas veces & 28.5 & 38.0 \\
3 & Algunas veces sí, algunas veces no & 34.4 & 72.4 \\
4 & Rara vez & 19.5 & 91.9 \\
5 & Nunca & 8.1 & 100.0 \\
& Total & 100.0 & \\
\hline
\end{tabular}

Se observa que un $18.2 \%$ de los estudiantes utilizan los servicios financieros que se ofrecen en la ciudad, un porcentaje alto (53.1\%) nunca o muy pocas veces los han utilizado. Para demostrar que estos porcentajes son significativo, se comprobó por medio de la prueba $t$ la hipótesis de investigación siguiente: Ho: Los estudiantes de nivel superior de Tuxtepec Oaxaca no usan los servicios financieros que ofrecen las Instituciones Bancarias.

Considerando el valor teórico de la media es 3.0, los resultados de la prueba t indican que hay una diferencia de -1.20 entre la media población y la media de la muestra, además el valor de significancia $(0.00)$ es menor de 0.05 , lo que indica que hay que rechazar la hipótesis nula y se concluye que los estudiantes usan los servicio financieros que ofrecen las instituciones financieras.

$$
\begin{aligned}
& H_{o} \geq 2.0 \\
& H_{i} \leq 2.0
\end{aligned}
$$

\begin{tabular}{|c|c|c|c|c|c|}
\hline $\mathrm{N}$ & Media & $\begin{array}{l}\text { Media } \\
\text { Teórica }\end{array}$ & $\begin{array}{l}\text { Desviación } \\
\text { estándar }\end{array}$ & \multicolumn{2}{|c|}{ Media de error estándar } \\
\hline 803 & 2.88 & 3.0 & 1.084 & \multicolumn{2}{|l|}{.038} \\
\hline \multicolumn{6}{|c|}{ Prueba de muestra única } \\
\hline \multicolumn{6}{|c|}{ Valor de prueba $=4.5$} \\
\hline \multirow[t]{2}{*}{$t$} & \multirow[t]{2}{*}{ gl } & \multirow{2}{*}{$\begin{array}{c}\text { Sig. } \\
\text { (bilateral) }\end{array}$} & & \multicolumn{2}{|c|}{$\begin{array}{l}\text { 95\% de intervalo de confianza de } \\
\text { diferencia }\end{array}$} \\
\hline & & & medias & Inferior & Superior \\
\hline-42.248 & 799 & .000 & -1.618 & -1.69 & -1.54 \\
\hline
\end{tabular}

Tabla 4 Estadísticas de muestra única

Finalmente la tabla señala que a un nivel de confianza de $95 \%$ el nivel de acceso a los servicios financieros está comprendido entre 2.88 y 2.81 . 


\section{CONCLUSIONES}

La inclusión financiera debe tener en cuenta el perfil de los estudiantes y si recurren a los servicios financieros que ofrecen las instituciones bancarias, con el fin de abordar soluciones específicas dirigidas a este sector.

Las evidencias de este estudio permiten afirmar que los estudiantes del nivel superior de Tuxtepec Oaxaca, usan los servicios financieros. Se puede observar que aun cuando es un porcentaje bajo (18.2\%) el que hace uso de ellos, este valor es significativo a 0.05 . Los resultados son coherentes con los reportados por las diferentes instituciones (UNAM, BANAMEX; 2008) que dan a conocer que los jóvenes operan algunos de los servicios que ofrecen las instituciones bancarias.

Sin embargo, es importante reiterar que existe un porcentaje del $8 \%$ de estudiantes que no recurren al sistema financiero, siendo este proporción una área de oportunidad para el proceso de inclusión financiera en México, dado que la inclusión financiera contribuye al desarrollo económico y favorece a la población; al permitir a los individuos tomar decisiones sobre la administración de su dinero que puede efectuarse a través del al ahorro, inversiones, transacciones, estas acciones promueve el bienestar para los individuos.

Pero sin la información necesaria, los individuos no tienen el conocimiento y por ende no pueden manejar sus recursos ni los procesos que se requieren para tener acceso a los productos y servicios financieros que promueven las instituciones bancarias. Es importante mencionar que un sector clave son los estudiantes de nivel superior para capacitarlos y evitar la exclusión financiera sobre todo en esta área del país, donde se requiere de gran desarrollo económico.

\section{REFERENCIAS}

AFI (2010). La medición de la inclusión financiera para entes reguladores: Diseño e implementación de encuestas. Recuperado de http://www.afiglobal.org/sites/default/files/pdfimages/afi\%20policy\%20paper-SP\%20(2).pdf

AFI (2013). Ubicando la inclusión financiera en el mapa mundial. México: Informe sobre avances de la Declaración Maya. Alianza para la Inclusión Financiera 5-8.

BANAMEX-UNAM, (2008). Primera encuesta de cultura financiera en México, Encuesta (en línea). Recuperado de https://www.banamex.com/demos/saber_cuenta/pdf/encuesta_corta_final.pdf.

Billimoria, J., Penner, J., \& Knoote, F. (2013). Developing the next generation of economic citizens: financial inclusion and education for children and youth. Enterprise Development \& Microfinance, 24(3), 204-217.

Billimoria, J., Penner, J., \& Knoote, F. (2013). Developing the next generation of economic citizens: financial inclusion and education for children and youth. Enterprise Development \& Microfinance, 24(3), 204-217.

Comisión Nacional Bancaria y de Valores (2015). Información estadística. México. Recuperado de http://www.cnbv.gob.mx/Paginas/PortafolioDelnformacion.aspx

Comisión Nacional Bancaria y de Valores (2015). Información estadística. México. Recuperado de http://portafoliodeinformacion.cnbv.gob.mx/. 
Comisión Nacional Bancaria y de Valores (2015). Información estadística. México. Recuperado de http://www.cnbv.gob.mx/Paginas/PortafolioDelnformacion.aspx

Comisión Nacional Bancaria y de Valores (2015). Información estadística. México. Recuperado de http://portafoliodeinformacion.cnbv.gob.mx.

Comisión Nacional para la Protección y Defensa de los Usuarios de Servicios Financieros (2010), Informe de la Tercera Semana Nacional de Educación Financiera, realizado por la Condusef, Octubre 2010.

Comisión Nacional para la Protección y Defensa de los Usuarios de Servicios Financieros (2010). Informe de la Tercera Semana Nacional de Educación Financiera, realizado por la Condusef.

CYFI Model of Economic Citizenship (2015). Children, Youth \& Finance https://issuu.com/childfinanceinternational/docs/children_youth__finance_2015/1?e=7 $128000 / 38088825$

Encuesta Nacional de Inclusión Financiera (2015). Boletín de Prensa Núm. 262/16. Recuperado de http://www.inegi.org.mx/saladeprensa/boletines/2016/especiales/especiales2016_06_0 2.pdf.

Encuesta Nacional de Inclusión Financiera (2015). Boletín de Prensa Núm. 262/16. Recuperado de http://www.inegi.org.mx/saladeprensa/boletines/2016/especiales/especiales2016_06_0 2.pdf

Herrera, E. Raccanello, K, (2014). Educación e inclusión financiera. Revista Latinoamericana de Estudios Educativos, 44(2), 119-14.

Johnson, E., \& Sherraden, M.S. (2007). From financial literacy to financial capability among youth. Journal of Sociology and Social Welfare 34(3), 119-145.

Lee, (2011). Workshop on Financial Inclusion Indicators. Recuperado de ww.bis.org/ifc/publ/ifcb38.pdf

Leeladhar. V. (2006). Taking Banking Services to the Common Man Financial Inclusion, Reserve Bank of India Bulletin. Retrieved from: http://rbidocs.rbi.org.in/rdocs/ Bulletin /PDFs/68236.pdf.

Lusardi, A. \& Mitchell 0., (2006). "Financial Literacy and Planning: Implication for Retirement Wellbeing", Working Paper, Pension Research Council, Wharton School, University of Pennsylvania. Recuperado de http://www.econ.yale.edu/ shiller/behmacro/200511/lusardi.pdf.

Máxima, C. (2016).Segundo Foro Internacional de Inclusión Financiera. Foro realizado en la Ciudad de México. Recuperado de https://www.gob.mx/forodeinclusionfinanciera.

Reddy, R., Bruhn, M. \& Tan, C. (2012). Capacidades financieras en México: resultados de la encuesta nacional sobre comportamientos, actitudes y conocimientos financieros. Banco Mundial, 82134, 1-94.

Sánchez, M. (2013). La tarea compartida de la inclusión financiera. $6^{\circ}$ Encuentro de Educación Financiera, Banamex.

Sherraden, M,and et.al.(2015). Financial Capability and Asset Building for All. American Academy of Social Work and Social Welfare, 1-29 